\title{
Cost- and energy-efficient backhaul options for heterogeneous mobile network deployments
}

\author{
Fabricio Farias $^{1} \cdot$ Matteo Fiorani $^{2} \cdot$ Sibel Tombaz $^{2} \cdot$ Mozhgan Mahloo $^{2} \cdot$ \\ Lena Wosinska $^{2}$ - Joao C. W. A. Costa ${ }^{1}$ - Paolo Monti ${ }^{2}(0)$
}

Received: 30 April 2016 / Accepted: 7 November 2016 / Published online: 22 November 2016

(c) The Author(s) 2016. This article is published with open access at Springerlink.com

\begin{abstract}
Heterogeneous networks (HetNets) have the potential to cater for the capacity requirements of mobile broadband services at reduced cost and energy consumption levels. One key aspect in HetNets is the role of the backhaul. More specifically, it is crucial for a mobile operator to understand the impact of specific technological and architectural upgrades in the mobile backhaul network on the capital and operational expenditure (i.e., CAPEX and OPEX). This paper proposes a comprehensive methodology that can be used to analyze the total cost of ownership of a number of backhaul options based on fiber, microwave, and copper technologies. The study considers both a Greenfield and a Brownfield scenario and takes into account the mobile broadband capacity requirements for the time period between years 2015 and 2025. From the results presented in the paper it can be concluded that even though microwave and fiber will be predominately used in the future, the possible migration paths leading to such fiber- and microwave-based backhaul scenarios might be different, depending upon factors such as spectrum and license costs, time to deployment, availability of equipment, and required quality of service levels.
\end{abstract}

This work has been supported by EIT Digital as part of the project "The Royal Gardens Case," by VINNOVA as part of the project

"Smart City Concepts in Curitiba: Innovation for Mobility and Energy Efficiency," and by Conselho Nacional de Desenvolvimento Cientifico e Tecnologico (CNPQ) and Coordenacao de Aperfeicoamento de Pessoal de Nivel Superior (CAPES).

$\triangle$ Paolo Monti

pmonti@kth.se

Fabricio Farias

fabriciosf@ufpa.br

1 Federal University of Para, Belém, Brazil

2 KTH Royal Institute of Technology, Kista, Sweden
Keywords Mobile broadband $\cdot$ Small cells $\cdot$ Heterogeneous networks (HetNet) - Backhaul - Total cost of ownership (TCO) - Greenfield deployment · Brownfield deployment . Migration strategies

\section{Introduction}

Heterogeneous network (HetNet) deployments are becoming more and more popular among mobile operators. The key rationale behind a HetNet deployment is to provide user coverage via macro base stations (BSs) and to guarantee high capacity to costumers only where needed via the ad hoc deployment of small cells, i.e., micro, pico, and/or femto BSs. HetNets have a number of advantages, which include reduced energy consumption both in indoor [1] and in outdoor [2] deployments. In fact, small cells consume less power than macro BSs. In addition, by using small cells to cater for capacity hot spots and to serve costumers residing indoors it is possible to reduce both the number of macro BSs needed in a given area and their transmission power, i.e., by serving mainly outdoors users macro BSs do not need to transmit anymore at very high power to penetrate walls to reach indoor customers.

The advantages of HetNet deployments come at the expense of a more complex and (possibly) more costly backhaul network, compared to the case of homogeneous mobile networks. For example, it has been shown that the total power consumption of a HetNet deployment to a large degree is affected not only by the presence of the backhaul [2] but also by its specific technological and architectural choices [3]. This means that backhaul networks take a share of the cost and of the energy consumption of mobile networks. How significant is this impact depends on the BS deployment scenario as well as on the technology and architectural choice for 
the backhaul itself. Currently backhaul networks are largely based on microwave, copper, and fiber. These technologies offer different advantages depending on the deployment scenario [4]. Fiber-based alternatives have a relatively high deployment cost (CAPEX) but provide long-term support in terms of offered capacity. A microwave-based backhaul is attractive in terms of short time to market, low investment in infrastructure, and simple deployment. Digital Subscriber Lines (DSLs) might be still appealing for backhauling indoor small cells in the presence of an existing copper infrastructure, bearing in mind their capacity limitations. All the above aspects become crucial for mobile operators the moment they need to make decisions on when and how to deploy their backhaul infrastructure. More specifically, operators need to understand in advance what will be the cost and the energy consumption impact of each one of these technologies (i.e., fiber, microwave, and copper) when having to deploy a completely new backhaul segment (i.e., Greenfield) or when having to upgrade an already existing one (i.e., Brownfield).

This paper presents a comprehensive methodology that can be used to analyze the total cost of ownership (TCO) of a number of backhaul network options based on fiber, copper, and microwave. The use case under examination considers an European urban scenario with both outdoor and indoor users. The latter are served by a layer of femto cells deployed inside the buildings where they reside, while the former are catered by macro BSs. The mobile broadband traffic levels considered in the study include values derived from the past 5 years (2010-2015) and also a short-to-mediumterm future outlook, i.e., requirement envisioned for the year 2016-2025. In order to consider a wide range of options, the TCO analysis presented in the paper looks at both Greenfield and Brownfield scenarios, and it also considers the case in which a new fiber-based backhaul segment can be either deployed (i.e., fiber trenching option) or rented as needed from a third-party entity (i.e., fiber leasing option). To the best of our knowledge this work is among the first presenting such a detailed TCO analysis where both Greenfield and Brownfield deployments are considered. The results derived from the study highlight that it is not possible to find a "one size fits all" backhaul solution. Even if there are no doubts that both microwave and fiber will play a predominant role in future backhaul networks, the possible migration paths might vary. This can be attributed to a number of factors, such as the presence of an existing infrastructure, spectrum and license costs, the availability of equipment, the degree of willingness to invest in a completely new infrastructure, the technological development (possibly reducing cost and power consumption of some equipment), and the quality of service (QoS) to be provided to the end-user. For example, in a Greenfield deployment fiber-based architectures are the most energy-efficient option. However, they lead to relatively high TCO values over a period of 15 years. Leasing the fiber infrastructure might help in reducing CAPEX, but if a mobile operator does not have the possibility to lease dark fibers, the use of a microwave-based backhaul can still provide low TCO values. On the other hand a microwavebased backhaul is not the best in terms of energy efficiency. In a Brownfield deployment, to maximize energy efficiency the best option is to replace immediately all the copper and microwave infrastructure with a fiber-based one. However, this requires a large investment that might not be fully compensated over a 15 -year period. In fact, the migration option that leads to the lowest TCO value is based on a gradual migration take-up, where the existing copper and microwave equipment is progressively replaced with fibers.

\section{Related work}

The first set of papers addressing the design of backhaul architectures for HetNets, i.e., $[3,5,6]$, focused mostly on energy consumption evaluation.

The authors in [5] assessed the impact of the backhaul segment on the overall HetNet energy consumption under different wireless traffic levels and considering different backhaul technologies. The results presented in the paper showed that the backhaul network can play a significant role in the total power consumption of a HetNet and, more importantly, that this role becomes even more prominent with increasing values of the wireless traffic. The authors in [3] provided models to compute the power consumed by a backhaul network based on fiber (with point-to-point connections) and microwave (with point-to-point, ring, and star topologies). The results presented in the paper showed how the backhaul network is responsible for a significant part of the overall power consumption of HetNets. The authors also showed that fiber-based backhaul architectures are significantly more energy efficient than their microwave-based counterparts. Finally, the authors in [6] analyzed several backhaul options for indoor small cell based on copper, fiber, and microwave. The objective was to evaluate whether backhaul might become a bottleneck when providing mobile broadband services to indoor users. The results presented in the paper showed that the backhaul power consumption may become an issue in the case of very dense indoor small cell deployments and they highlighted how optical fiber is the most energy-efficient technology option.

While the papers just mentioned mainly focused on energy consumption modeling and estimations, there are other works that has been recently published whose aim is to assess and minimize the TCO of backhaul networks. The authors in [7] presented a technoeconomic model to calculate the TCO of HetNets that accounts for the cost of both the wireless (i.e., macro and femto BSs) and the backhaul infrastructure. The results presented in the paper show that in urban areas it is 
possible to reach up to $70 \%$ cost savings using indoor small cell deployments in place of traditional ones based on macro BSs. The works in $[8,9]$ studied both homogenous and heterogeneous wireless architectures and assessed the impact of their backhaul network on the entire TCO. The results indicate that in HetNets the backhaul network represents a considerable portion of the TCO, while in homogeneous networks, based on macro BSs only, the backhaul cost does not affect significantly the TCO. In [10] the authors evaluated a microwave-based backhaul network for small cells in terms of cost-efficiency and time required for deployment. Their results showed that point-to-point microwave is a costefficient technology to provide high backhaul capacity in short deployment times. Moreover, the authors in $[4,8,11,12]$ assessed the Capital Expenditure (CAPEX) and Operational Expenditure (OPEX) of mobile backhaul networks in several Greenfield deployment scenarios. More specifically, paper [8] assessed the economic impact of fiber and microwave backhaul solutions in the presence of low and high wireless capacity demands, while paper [11] compared the cost of different backhaul deployments based on fiber and copper in a scenario where fixed broadband access is already provided using fiber to the node solutions. Both papers considered HetNets based on small cell deployments. These results show that copper-based backhaul networks can cost up to $70 \%$ less than fiber-based backhaul networks. Finally, papers $[4,12]$ presented a TCO model for backhaul networks that includes a detailed CAPEX and OPEX breakdown. These studies concluded that there is a significant impact in terms of CAPEX during the first years of the wireless network deployments. However, these costs can lead to a good return of investment in the following years when the penetration rate of small cells increases.

To the best of our knowledge, the literature mainly considers Greenfield deployment scenarios where the existence of an infrastructure that can be potentially re-used to backhaul small cells is not accounted for. On the other hand, the majority of the households today are connected via copper cables used to provide Digital Subscriber Line (DSL) services. DSL is a very popular last-mile fixed broadband technology, and up to 2011, about three-fourth of the FTTX (fiber to the $\mathrm{x}$ $=$ node/cabinet/building) solutions relied on DSL to connect to end-users [13]. In such a scenario it might become economically convenient to re-use as much as possible legacy DSL solutions for backhauling femto cells, especially the ones deployed indoors. We refer to these cases as Brownfield backhaul deployments.

The contribution of this paper is twofold. It combines the work of [6] and [4] by analyzing both the power consumption and the TCO value of different backhaul architectures in a Greenfield scenario. This allows new important considerations, with respect to the ones already discussed in [6] and [4]. Second, this paper presents and compares the power con- sumption and the TCO value of different migration options for backhaul networks in a number of Brownfield deployment scenarios. This is a new and important contribution because it shows how different migration paths can lead to very different power consumption levels and TCO values. These new results can help mobile network operators to understand: (i) which is the best way to deploy/upgrade their current backhaul network infrastructure, and (ii) how to choose (when needed) the most energy- and cost-efficient migration paths.

\section{TCO assessment methodology}

This section provides an overview of the proposed methodology for assessing the TCO of mobile backhaul networks. The methodology is divided into four phases (Fig. 1).

The first phase is the traffic demand estimation. It is used to compute the expected wireless traffic requirements in the deployment area for a given year. The inputs are the population density and other relevant data describing the network and service usage such as: the number of active mobile subscribers, the user profile (e.g., heavy or ordinary), the daily traffic variation, and the penetration rate of different mobile terminals. Using the long-term large-scale traffic model proposed in [6] this phase returns the value of the area traffic demand.

The second phase is the wireless deployment. It is used to determine the number of BSs to be deployed in the area. In this case the inputs are the area traffic demand from the

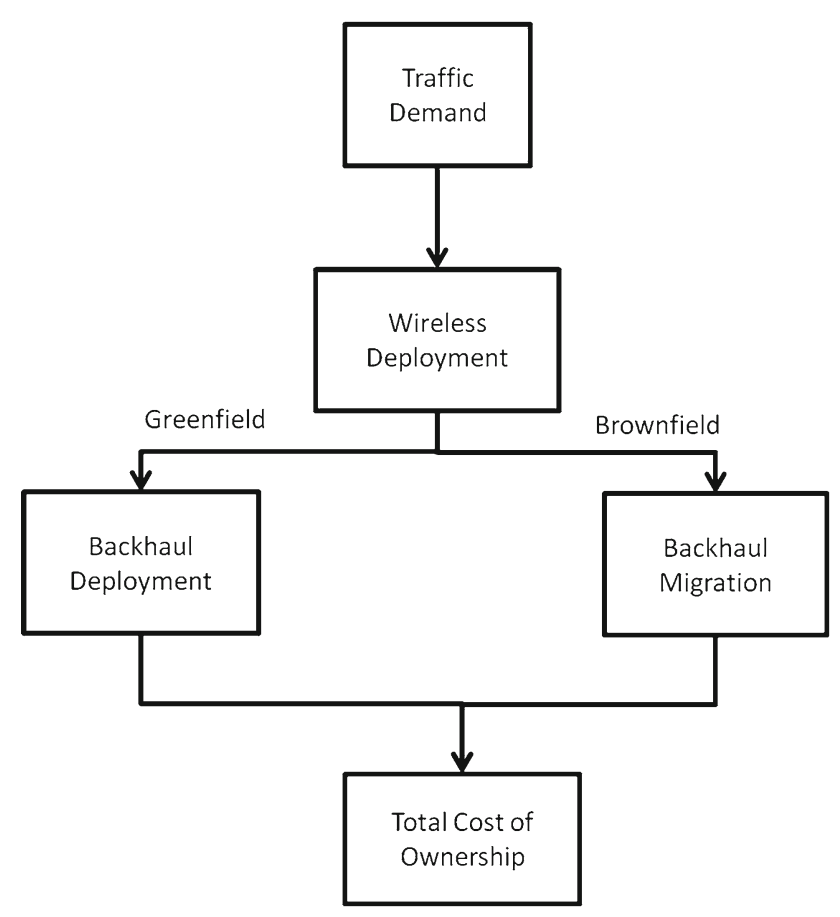

Fig. 1 TCO assessment methodology 
previous step in addition to other parameters related to the wireless network technologies to be used, i.e., the capacity of all BS types and the penetration rate of the small cells [6]. Accordingly, it is possible to obtain the number of macro BSs and small cells required to cover the area and to satisfy the wireless traffic requirements of a given year.

The third phase can be of two types. The first one called backhaul deployment refers to a Greenfield scenario where no pre-existing backhaul infrastructure is present in the area. The second one called backhaul migration refers to a Brownfield scenario where there is a pre-existing infrastructure that can be re-used for backhauling small cells. For the backhaul deployment case the inputs are: the output of the wireless network deployment step, the peak capacity of both macro BSs and small cells, the backhaul architecture, and the transmission/switching capacity of the backhaul equipment. For the backhaul migration case there is an additional input, i.e., the description of the technical details of the already existing infrastructure that can be used for backhauling small cells. For both options the output is the number of network devices (e.g., microwave antennas, optical transceivers, and DSL modems) that will have to be deployed in the area.

Finally, the last phase is the total cost of ownership evaluation that returns the overall TCO of the backhaul network. Besides the output of the previous phase, the inputs for this step are: the cost of the equipment in the backhaul network and the cost of other related assets (e.g., energy consumption, fiber trenching, and spectrum leasing). The details of the model utilized to evaluate the total TCO of the backhaul network (which includes both CAPEX and OPEX) are provided in the next section.

\section{TCO assessment model}

We provide a detailed description of the TCO assessment methodology introduced in the previous section, and we present the analytic models adopted in our study. First we describe in details the traffic estimation model, then we present the wireless and backhaul network deployment strategy, and we conclude with the detailed description of the TCO evaluation model. The notations used in this section are summarized in Table 1.

\subsection{Traffic demand estimation}

In this paper we use the traffic model presented in [6] and [14] to estimate the area traffic demand for a given year. Firstly, we calculate the daily area traffic demand $R(t)$ as:

$R(t)=\frac{\rho \alpha(t)}{N_{\mathrm{op}}} \sum_{k} r_{k} s_{k}$,
Table 1 Notation used in the paper

\begin{tabular}{|c|c|}
\hline Notation & Description \\
\hline$\alpha(t)$ & $\begin{array}{l}\text { Percentage of active mobile users at a given time } \\
\text { of the day } t\end{array}$ \\
\hline$\alpha_{\max }$ & Peak daily percentage of active mobile users \\
\hline$\rho$ & Population density in the area \\
\hline$\eta$ & Femto penetration rate \\
\hline$\tau$ & Peak daily area traffic demand \\
\hline$r$ & Average data rate per active user \\
\hline$r_{k}$ & Average data rate for terminal type $k$ \\
\hline$r_{k}^{\text {heavy }}$ & Average data rate generated by a heavy user \\
\hline$r_{k}^{\text {ordinary }}$ & Average data rate generated by an ordinary user \\
\hline$R(t)$ & Daily area traffic demand \\
\hline$s_{k}$ & Fraction of the subscribers using terminal type $k$ \\
\hline$A$ & Area under consideration \\
\hline$A g g_{\text {tot }}$ & $\begin{array}{l}\text { Aggregated traffic collected at a Carrier Ethernet } \\
\text { Switches }\end{array}$ \\
\hline$C_{\text {maxmacro }}$ & Maximum transmission capacity of a macro BS \\
\hline$h \%$ & $\begin{array}{l}\text { Percentage of subscribers classified as heavy } \\
\text { users }\end{array}$ \\
\hline$k$ & Terminal type \\
\hline $\operatorname{Max}_{\text {Trans/Link }}$ & Maximum transmission rate per uplink interface \\
\hline$N_{\text {ap }}$ & Total number of apartments \\
\hline$N_{\mathrm{b}}$ & Number of buildings \\
\hline$N_{\text {bf }}$ & Number of buildings with femto BSs \\
\hline$N_{\text {DSLAM }}$ & Number of DSLAMs \\
\hline$n_{\text {ports }}^{\text {DSLAM }}$ & Number of ports per DSLAM \\
\hline$N$ femto & Number of deployed femto BSs \\
\hline$N_{\mathrm{s}}^{\mathrm{F}}$ & Number of Carrier Ethernet Switches \\
\hline$N_{\mathrm{ul}}^{\mathrm{F}}$ & $\begin{array}{l}\text { Number of uplink connections toward Carrier } \\
\text { Ethernet Switches and Metro Network }\end{array}$ \\
\hline$n_{\text {ports }}^{\mathrm{F}}$ & Number of ports of a Carrier Ethernet Switches \\
\hline$n_{\text {ports }}^{\mathrm{GES}}$ & Total number of ports of the GES \\
\hline$N_{\text {hub }}$ & Number of hubs \\
\hline$N_{\text {macro }}$ & Total number of macro base stations \\
\hline$N_{\mathrm{s}}^{\mathrm{MW}}$ & $\begin{array}{l}\text { Total number of Carrier Ethernet Switches inside } \\
\text { the hubs }\end{array}$ \\
\hline$N_{\mathrm{ul}}^{\mathrm{MW}}$ & $\begin{array}{l}\text { Total number of uplink connections between the } \\
\text { Carrier Ethernet Switches and MN }\end{array}$ \\
\hline$n_{\max }^{\text {MWlink }}$ & Maximum number of links supported by a hub \\
\hline$N_{\mathrm{OLT}}$ & Number of OLTs in the network \\
\hline$n_{\text {cards }}^{\text {OLT }}$ & Number of OLT cards \\
\hline$N_{\text {op }}$ & Number of mobile operators serving the area \\
\hline$n_{\text {ports }}^{\text {Spliter }}$ & Number of ports per passive splitter \\
\hline$N_{\text {useractive/macro }}$ & $\begin{array}{l}\text { Maximum number of active users that can be } \\
\text { served by a macro BS }\end{array}$ \\
\hline$P_{\mathrm{bh}}^{\mathrm{arch} 1}$ & Power consumption of Architecture 1 \\
\hline$P_{\mathrm{bh}}^{\operatorname{arch} 2}$ & Power consumption of Architecture 2 \\
\hline$P_{\mathrm{bh}}^{\operatorname{arch} 3}$ & Power consumption of Architecture 3 \\
\hline
\end{tabular}


Table 1 continued

\begin{tabular}{ll}
\hline Notation & Description \\
\hline$P_{\mathrm{bh}}^{\text {arch }}$ & Power consumption of Architecture 4 \\
$P_{\mathrm{bh}}^{\text {arch }}$ & Power consumption of Architecture 5 \\
$P_{\mathrm{DSLAM}}$ & Power consumption of a DSLAM \\
$P_{\text {femto }}$ & Power consumption of a femto BS \\
$P_{\mathrm{GES}}$ & Power consumption of a GES \\
$P_{\text {high-c }}$ & Power consumption of a microwave antenna in \\
& high capacity mode \\
$P_{\text {low-c }}$ & Power consumption of a microwave antenna in \\
$P_{\mathrm{modem}}$ & low capacity mode \\
$P_{\mathrm{GES}}^{\mathrm{max}}$ & Power consumption of a VDSL2 modem \\
$P_{\mathrm{OLT}}$ & Maximum power consumption of a GES \\
$P_{\mathrm{ONU}}$ & Power consumption of an OLT \\
$P_{\mathrm{s}}^{\mathrm{F}}$ & Power consumption of an ONU \\
$P_{\mathrm{s}}^{\mathrm{MW}}$ & Power consumption of a Carrier Ethernet Switch \\
$P_{\mathrm{SFP}}$ & Power consumption of a Carrier Ethernet Switch \\
$P_{\mathrm{SFP}+}$ & Power consumption of a SFP \\
\hline
\end{tabular}

where $\rho$ represents the population density in the area and $\alpha(t)$ represents the percentage of active mobile users at a given time of the day $t$. The term $k$ indicates the terminal type, and $N_{\text {op }}$ represents the number of mobile operators serving the area. Finally, $r_{k}$ and $s_{k}$ represent the average data rate for terminal type $k$ and the fraction of the subscribers using terminal type $k$, respectively. We consider three different terminal types, i.e., laptop, tablet, and smartphone [14]. The users are divided into two groups, i.e., heavy and ordinary users, where the capacity requirements of an ordinary user are lower than those of a heavy user [14]. Based on the assumption that $h \%$ of the subscribers are classified as heavy users, the average data rate generated by terminal type $k$ can be defined as:

$r_{k}=\frac{h \cdot r_{k}^{\text {heavy }}+(100-h) r_{k}^{\text {ordinary }}}{100},[\mathrm{Mbps}]$

where $r_{k}^{\text {heavy }}$ [Mbps] and $r_{k}^{\text {ordinary }}$ [Mbps] represent the average data rate generated by a heavy and an ordinary user, respectively.

Using Eqs. (1) and (2) in combination with the forecasted values of the other parameters (i.e., $h, N_{\mathrm{op}}, s_{k}, r_{k}^{\text {heavy }}$, and $r_{k}^{\text {ordinary }}$ ) it is possible to calculate the daily area traffic demand for a given year. In our study we design the wireless and backhaul networks in order to support the peak daily area traffic demand $\tau\left(\mathrm{Mbps} / \mathrm{km}^{2}\right)=\max _{t}(R(t))$, which corresponds to the case $\alpha(t)=\alpha_{\max }$.

\subsection{Wireless deployment}

There are several possible choices for which wireless network architectures could be deployed to satisfy the area traffic demand. They depend on the combination of BS type used (e.g., macro, micro, pico, and femto). In this paper we consider a wireless network architecture that consists of both outdoor macro and indoor femto BSs. We assume that the macro BSs are deployed by the network operator at the rooftops, while the femto BSs are deployed by the end-users in their apartments.

The number of deployed femto BSs $\left(N_{\text {femto }}\right)$ is given as a function of the femto penetration rate $(\eta)$ and the total number of apartments $\left(N_{\text {ap }}\right)$ in the area: $N_{\text {femto }}=N_{\text {ap }} \eta$. It is assumed that the apartments are uniformly distributed in $N_{\mathrm{b}}$ buildings. Since the macro cellular network must serve the remaining active users (i.e., those which are not served by the femto BSs), the required number of macro BSs can be computed as:

$N_{\text {macro }}=\frac{\rho A(1-\eta) \alpha_{\max }}{N_{\text {useractive }} / \text { macro }}$,

where $A\left[\mathrm{~km}^{2}\right]$ represents the area under consideration.

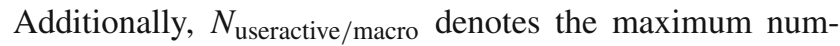
ber of active users that can be served by a macro BS, i.e., $N_{\text {useractive/macro }}=\frac{C_{\text {maxmacro }}}{r}$, which is the ratio between the maximum transmission capacity of a macro $\mathrm{BS}\left(C_{\text {maxmacro }}\right)$ and the average data rate per active user $(r)$.

\subsection{Backhaul architectures}

This section provides a detailed description of the backhaul architectures analyzed in the paper. We consider five backhaul architectures (Fig. 2): (i) Microwave, (ii) Fiber to the Node + Microwave, (iii) Fiber to the Building + Microwave, (iv) Fiber to the Home+Microwave, and (v) Fiber to the Home.

\subsubsection{Architecture 1 (A1): Microwave}

The first backhaul architecture is shown in Fig. 2a and is based on a microwave network with a star topology [3]. The outdoor macro BSs are equipped with microwave antennas, which are directly connected to a hub using a dedicated microwave link. Meanwhile, the femto BSs inside a building are connected to a Gigabit Ethernet Switch (GES) using copper cables (e.g., CAT 5/6/7). The GES aggregates the traffic from the femto BSs inside a building and provides connectivity to a microwave antenna placed on the rooftop. The antenna is in turn connected to a hub using a dedicated microwave link. The microwave links can operate for instance in the 
(a)

ARCHITECTURE 1 (b) (c) (d) (e)

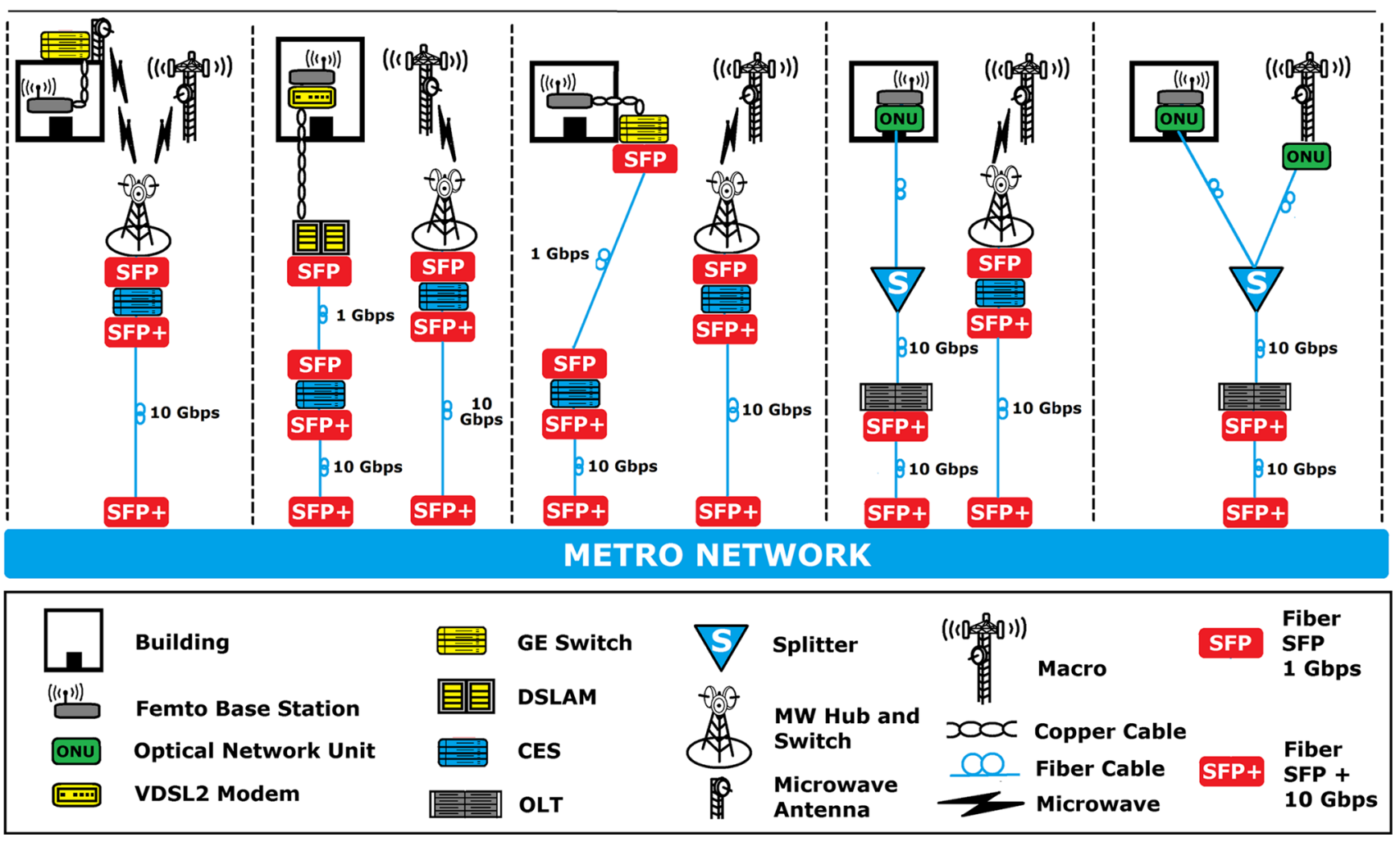

Fig. 2 Considered backhaul architectures. a Microwave. b Fiber to the Node +Microwave. c Fiber to the Building + Microwave. d Fiber to the Home + Microwave. e Fiber to the Home

range between 5 and $80 \mathrm{GHz}$, which is the most suited for urban areas [15]. The hubs are equipped with Carrier Ethernet Switches (CESs) that are responsible to aggregate the traffic from the microwave antennas and send it toward the Metro Network (MN). The transmission toward the MN is performed using optical point-to-point links. Small form-factor pluggable plus (SFP+) transceivers are used for transmitting and receiving the optical signals.

The power consumption of this architecture, i.e., $P_{b h}^{\mathrm{arch} 1}$, can be expressed as:

$$
\begin{aligned}
P_{\mathrm{bh}}^{\text {arch } 1}= & \left(N_{\mathrm{bf}}+N_{\text {macro }}\right) P_{\text {low-c }}+N_{\mathrm{bf}} P_{\mathrm{GES}} \\
& +N_{\text {hub }} P_{\text {high-c }}+2 N_{\mathrm{ul}}^{\mathrm{MW}} P_{\mathrm{SFP}+} \\
& +N_{\mathrm{s}}^{\mathrm{MW}} P_{\mathrm{s}}^{\mathrm{MW}},
\end{aligned}
$$

where $N_{\text {bf }}, N_{\text {macro }}, N_{\text {hub }}, N_{\mathrm{s}}^{\mathrm{MW}}$, and $N_{\mathrm{ul}}^{\mathrm{MW}}$ represent the number of buildings with femto BSs, the number of macro BSs, the number of hubs, the total number of CES inside the hubs, and total number of uplink connections between the CESs and MN, respectively. On the other hand, $P_{\text {low-c }}$ and $P_{\text {high-c }}$ represent the power consumption of a microwave antenna in low and high capacity mode, respectively (according to the power model presented in [3]). Finally, $P_{\mathrm{GES}}, P_{\mathrm{SFP}+}$, and
$P_{\mathrm{s}}^{\mathrm{MW}}$ denote the power consumption of a GES, of a SFP+, and of a CES, respectively. It can be observed that $N_{\text {hub }}$ is a function of the maximum number of links supported by a hub $\left(n_{\max }^{\text {MWlink }}\right)$, i.e., $N_{\text {hub }}=\left\lceil\frac{N_{\text {bf }}+N_{\text {macro }}}{n_{\text {max }}^{\text {MWlink }}}\right\rceil$. Finally, $N_{\mathrm{ul}}^{\mathrm{MW}}$ depends on the total aggregated traffic collected at the CES $\left(\mathrm{Agg}_{\text {tot }}\right)$ and on the maximum transmission rate per uplink interface $\left(\operatorname{Max}_{\text {Trans/Link }}\right)$. It can be computed according to the following equation:

$N_{\mathrm{ul}}^{\mathrm{MW}}=\max \left(N_{\mathrm{s}}^{\mathrm{MW}} ;\left\lceil\frac{\mathrm{Agg}_{\mathrm{tot}}}{\operatorname{Max}_{\text {Trans } / \text { Link }}}\right\rceil\right)$.

\subsubsection{Architecture 2 (A2): Fiber to the Node+Microwave}

The second backhaul solution, referred to as Fiber to the Node $($ FTTN $)+$ Microwave, is presented in Fig. 2b. It consists of a hybrid architecture that employs fiber and copper for backhauling indoor femto BSs and microwave for backhauling outdoor macro BSs. With this architecture, the femto BSs are backhauled using Very high-speed Digital Subscriber Line version 2 (VDSL2) links, which utilize a frequency range up to $30 \mathrm{MHz}$ and provide a maximum downlink capacity over copper cables of 100 [Mbps] for distances up to $300 \mathrm{~m}$. 
Each femto BS is connected to a VDSL2 modem that in turn is connected to a DSL Access Multiplexer (DSLAM) using a high-speed connection over copper. The DSLAM is located at a remote node that is usually placed inside a street cabinet close to the user premises. The DSLAMs are connected to a number of CESs using point-to-point optical links. Small form-factor pluggable (SFP) transceivers are used for transmitting and receiving the optical signal between the DSLAMs and the CESs. On the other hand, the macro BSs are connected to the CESs using microwave links (as it was already the case in A1). The CESs aggregate the traffic coming from the wireless network before sending it to the MN via optical links using SFP+ modules.

The power consumption of this second architecture, i.e., $P_{\mathrm{bh}}^{\mathrm{arch} 2}$, is obtained through the following formula:

$$
\begin{aligned}
P_{\mathrm{bh}}^{\mathrm{arch} 2}= & \left(P_{\text {femto }}+P_{\text {modem }}\right) N_{\text {femto }}+N_{\mathrm{s}}^{F} P_{\mathrm{s}}^{F} \\
& +\left(P_{\mathrm{DSLAM}}+2 P_{\mathrm{SFP}}\right) N_{\text {DSLAM }} \\
& +N_{\text {macro }} P_{\text {low-c }}+N_{\text {hub }} P_{\text {high-c }} \\
& +N_{\mathrm{s}}^{\mathrm{MW}} P_{\mathrm{s}}^{\mathrm{MW}}+2 P_{\mathrm{SFP}+}\left(N_{\mathrm{ul}}^{\mathrm{F}}+N_{\mathrm{ul}}^{\mathrm{MW}}\right),
\end{aligned}
$$

where $P_{\text {femto }}, P_{\text {modem }}, P_{\mathrm{DSLAM}}, P_{\mathrm{SFP}}$, and $P_{\mathrm{s}}^{\mathrm{F}}$ represent the power consumption of a femto BSs, of a VDSL2 modem, of a DSLAM, of a SFP, and of a CES, respectively. Moreover, $N_{\mathrm{DSLAM}}, N_{\mathrm{s}}^{\mathrm{F}}$, and $N_{\mathrm{ul}}^{\mathrm{F}}$ denote the number of DSLAMs, the number of CESs, and the number of uplink connections toward the MN. The value of $N_{\text {DSLAM }}$ is a function of the number of ports per DSLAM ( $\left.n_{\text {ports }}^{\text {DSLAM }}\right)$, i.e., $N_{D S L A M}=$ $\left\lceil\frac{N_{\text {femto }}}{n_{\text {ports }}^{\text {DSLAM }}}\right\rceil$. Similarly, the value of $N_{\mathrm{s}}^{\mathrm{F}}$ is a function of the number of ports of a CES $\left(n_{\text {ports }}^{\mathrm{F}}\right)$, i.e., $N_{\mathrm{s}}^{\mathrm{F}}=\left\lceil\frac{N_{\text {DSLAM }}}{n_{\text {ports }}^{\mathrm{F}}}\right\rceil$. The value of $N_{\text {hub }}$ is a function of the maximum number of links supported by a hub, i.e., $N_{\text {hub }}=\left\lceil\frac{N_{\text {macro }}}{n_{\max }^{\text {MWLink }}}\right\rceil$. Finally, the value of $N_{\mathrm{ul}}^{\mathrm{F}}$ can be computed as:

$N_{\mathrm{ul}}^{\mathrm{F}}=\max \left(N_{\mathrm{s}}^{\mathrm{F}} ;\left\lceil\frac{\mathrm{Agg}_{\mathrm{tot}}}{\operatorname{Max}_{\text {Trans/Link }}}\right\rceil\right)$.

\subsubsection{Architecture 3 (A3): Fiber to the Building + Microwave}

The third backhaul architecture, referred to as Fiber to the Building (FTTB)+ Microwave, is shown in Fig. 2c. Similarly to A1, the femto BSs inside a building are connected to a GES using copper cables. The GES is in turn connected to a CES using optical point-to-point links. SFP transceivers are used at the GES and at the CES to transmit and receive the optical signal. The CESs are connected to the MN using optical links and SFP+ transceivers. Moreover, the macro BSs are backhauled utilizing the previously described microwave infrastructure.

The power consumption of $\mathrm{A} 3$ can be computed using the following formula:

$$
\begin{aligned}
P_{\mathrm{bh}}^{\mathrm{arch} 3}= & \left(P_{\mathrm{GES}}+2 P_{\mathrm{SFP}}\right) N_{\mathrm{bf}}+N_{\mathrm{s}}^{F} P_{\mathrm{s}}^{F} \\
& +N_{\text {macro }} P_{\text {low-c }}+N_{\text {hub }} P_{\text {high-c }} \\
& +N_{\mathrm{s}}^{\mathrm{MW}} P_{\mathrm{s}}^{\mathrm{MW}}+2 P_{\mathrm{SFP}+}\left(N_{\mathrm{ul}}^{\mathrm{F}}+N_{u l}^{M W}\right),
\end{aligned}
$$

where the number of CES is $N_{\mathrm{s}}^{F}=\left\lceil\frac{N_{\mathrm{bf}}}{n_{\text {ports }}^{F}}\right\rceil$. Additionally, $N_{h u b}$ and $N_{\mathrm{s}}^{\mathrm{MW}}$ are computed in the same way as for the case of $\mathrm{A} 2$.

\subsubsection{Architecture 4 (A4): Fiber to the Home + Microwave}

The fourth backhaul solution, referred to as Fiber to the Home $($ FTTH $)+$ Microwave, is shown in Fig. 2d. In this architecture the indoor femto BSs are backhauled using passive optical networks (PONs). Each femto BS inside a building is directly connected to an optical network unit (ONU). The ONUs are then connected to optical line terminals (OLTs) through passive optical splitters. The OLTs are connected to the MN using optical links and SFP+ transceivers. On the other hand, the macro BSs are backhauled utilizing the same microwave network design presented for the previous architectures. The power consumption of A4 can be expressed as:

$$
\begin{aligned}
P_{\mathrm{bh}}^{\mathrm{arch} 4}= & N_{\text {femto }} P_{\mathrm{ONU}}+N_{\mathrm{OLT}} P_{\mathrm{OLT}} \\
& +N_{\text {macro }} P_{\text {low-c }}+N_{\text {hub }} P_{\text {high-c }} \\
& +N_{\mathrm{s}}^{\mathrm{MW}} P_{\mathrm{s}}^{\mathrm{MW}}+2 P_{\mathrm{SFP}+}\left(N_{\mathrm{ul}}^{\mathrm{F}}+N_{\mathrm{ul}}^{\mathrm{MW}}\right),
\end{aligned}
$$

where $P_{\mathrm{ONU}}$ and $P_{\mathrm{OLT}}$ represent the power consumption of an ONU and of an OLT, respectively. The number of ONUs $\left(N_{\mathrm{ONU}}\right)$ is equal to the number of femto BSs $\left(N_{\text {femto }}\right)$. Meanwhile, $N_{\text {OLT }}$ represents the number of OLTs in the network, which is a function of the number of ports per passive splitter $\left(n_{\text {ports }}^{\text {Spliter }}\right)$ and the number of OLT cards $\left(n_{\text {cards }}^{\text {OLT }}\right) . N_{\text {OLT }}$ can be obtained as:

$N_{\mathrm{OLT}}=\left\lceil\frac{N_{\mathrm{ONU}}}{n_{\text {ports }} n_{\text {cards }}^{\text {Splite }}}\right\rceil$.

Finally, the dimensioning of the microwave network for backhauling the outdoor macro BSs is the same as the one obtained for A2.

\subsubsection{Architecture 5 (A5): Fiber to the Home}

The last proposed backhaul solution, referred to as Fiber to the Home (FTTH) backhaul, is shown in Fig. 2e, and it is 


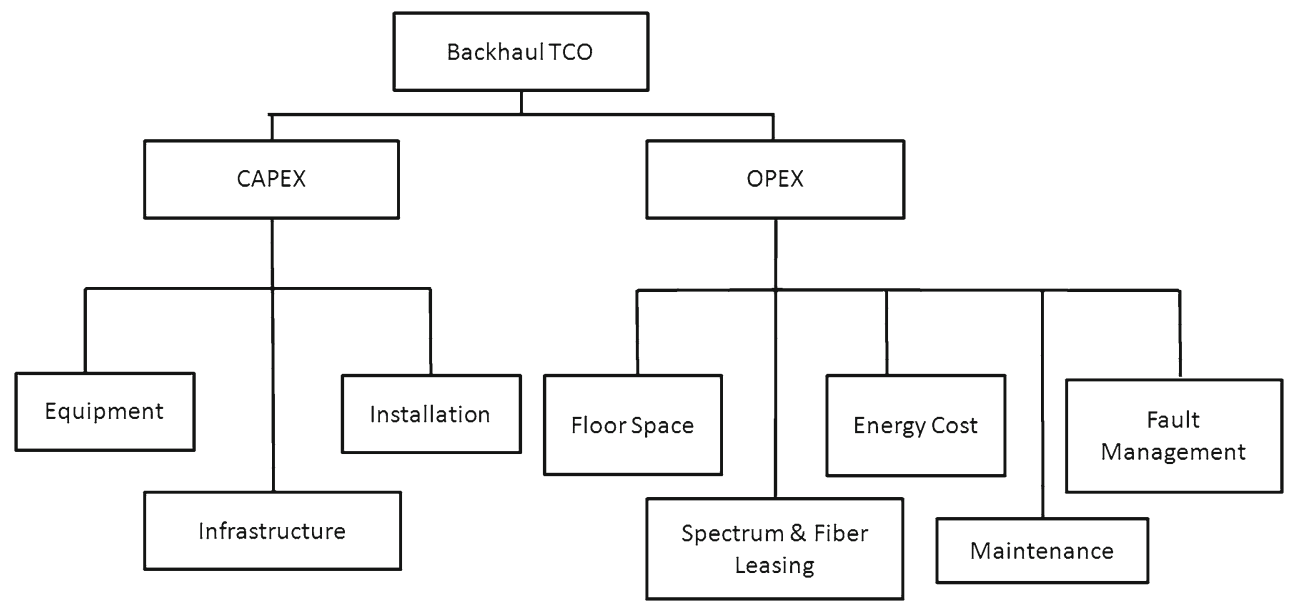

Fig. 3 Backhaul total cost of ownership model

based on PONs for backhauling both femto and macro BSs. Here, each one of them is equipped with an ONU connected to an OLT via a passive optical network. The power consumption of this architecture can be expressed as:

$$
\begin{aligned}
P_{\mathrm{bh}}^{\mathrm{arch} 5}= & \left(N_{\text {femto }}+N_{\text {macro }}\right) P_{\mathrm{ONU}} \\
& +N_{\mathrm{OLT}} P_{\mathrm{OLT}}+2 N_{\mathrm{ul}}^{\mathrm{F}} P_{\mathrm{SFP}+} .
\end{aligned}
$$

In this architecture the total number of ONUs corresponds to the total number of BSs (i.e., femto and macro) in the area. Moreover, $N_{\mathrm{OLT}}$ is calculated as in A4.

\subsection{TCO evaluation}

In this section we describe in detail the model used for evaluating the TCO of the backhaul architectures described in the previous section. The TCO model is depicted in Fig. 3 and includes both CAPEX and OPEX.

The CAPEX is obtained as the sum of the costs of the Infrastructure, Installation, and Equipment. Here, the Infrastructure cost refers to the fiber trenching costs (i.e., cost for trenching $1[\mathrm{~km}]$ of fiber times the total fiber length $[\mathrm{km}]$ required to serve the area). Furthermore, the Installation cost refers to the cost for installing and setting up the backhaul network equipment. This value is computed by multiplying the total time needed to install the network equipment [hours] with the salary of a technician [USD/hour]. Finally, the Equipment cost is the sum of all the backhaul network equipment costs.

The OPEX accounts for the yearly expenses related to the backhaul network operation. It is the sum of five contributions: Energy, Floor space, Spectrum and fiber leasing, Maintenance, and Fault management. The Energy cost is computed as the product of the cost of energy [USD/kWh] and the sum of the energy (i.e., expressed in $\mathrm{kWh}$ ) consumed by the entire backhaul network. The latter value is obtained using the power models in Sect. 4.3 and assuming that all the network equipment is active all the time. The Floor space cost is calculated by multiplying the total area required to house the backhaul equipment (e.g., expressed in square meters and including central offices, local exchanges, and antenna mounts) times the rent cost $\left[\mathrm{USD} / \mathrm{m}^{2}\right]$. The Spectrum and fiber leasing cost covers the expenses of leasing the microwave spectrum [USD/Link] and dark fibers [USD/km]. The Maintenance cost represents the expenditures related to monitoring, repairing, and testing network equipment at the central offices (CO), the local exchanges, and the microwave hubs. It also includes the yearly fees for the software licenses. Finally, the Fault management accounts for cost of repairing each failure that occurs in the backhaul network. More details about the TCO model for backhaul networks are available in the work presented in [4].

Before going into the details of the case study illustrated in the next section it is worth to highlight the following. The proposed four-step TCO assessment methodology is general and can be applied to any possible mobile network deployment scenario (i.e., the methodology is not tied to the use case specifically considered in this paper). On the other hand, the power models described so far are specific for the architectures under consideration, i.e., A1-5. However, these models can be easily adapted to evaluate the power consumption of any backhaul network based on the combination of copper, microwave, and fiber technologies. Finally, the TCO framework utilized to compute the network CAPEX and OPEX is general and can be applied to any backhaul network architecture.

\section{Case study}

In this section the methodology and power consumption models discussed so far are used to evaluate the TCO of 
the considered backhaul architectures in the context of an European urban scenario. It is assumed that the area under consideration is $A=100\left[\mathrm{~km}^{2}\right]$ with buildings uniformly distributed measuring $80 \times 80 \mathrm{~m}$ and separated from each other by $20 \mathrm{~m}$. The total number of buildings in the area is set to be 10,000, each one with 5 floors and 2 apartments per floor (i.e., the total number of apartments $N_{\text {ap }}$ corresponds to 100,000). In addition, it is assumed that the population density is $\rho=3000$ users $/ \mathrm{km}^{2}$ and that only one operator serves the area. Moreover, the distances between the $\mathrm{CO}$, local exchanges, and end-users are calculated using a Manhattan street model [16]. In order to compute the traffic demand $\tau$, we assume that $16 \%$ of the subscribers are active during peak hours (i.e., $\alpha_{\max }=16 \%$ ) and that the capacity requirement of an ordinary user is $1 / 8$ of the one of a heavy user [14]. Moreover, we assume that a laptop user generates two and eight times more data traffic than a tablet and a smartphone user, respectively. More details about the assumption used to compute the traffic demand can be found in $[14,17]$.

The study presented in the paper considers a 15-year time period that goes from year 2010 until year 2025 . For each year the traffic demand estimation phase described in Sect. 4.1 is used. It is assumed that in the year 2011 the area is served by a homogeneous wireless network based only on macro BSs, i.e., $\eta=0$. Starting from the year 2010, the wireless deployment evolves toward HetNets (i.e., with the gradual introduction of femto BSs) and there is a linear increase in the femto penetration rate of 5\% every year. Regarding the backhaul network, it is assumed that in 2010 the macro BSs are backhauled using microwave point-to-point links.

In the Greenfield scenario, starting from the year 2011, the operator deploys a new backhaul infrastructure to support HetNets. The new infrastructure can either be based on microwave or fiber (it is assumed that the operator will not deploy a new infrastructure based on copper because of its limited capability of supporting high capacity over long distances). As a result, in the Greenfield scenario, the operator (starting from year 2011) can choose either one of backhaul A1, A3, A4 and A5, described in Sect. 4.3.

On the other hand, in the Brownfield scenario the operator is able to leverage on the already existing fiber and copper infrastructure, i.e., the operator owns a backhaul infrastructure like the one described by A2. As a result, in the year 2011 the operator faces two options for network upgrade. The first choice is to continue deploying a microwave-based backhaul (i.e., as the one described by A1), while the second option is to migrate to new network architectures that reuse legacy ducts and trench infrastructure. This migration can be executed according to different models. In the following, we describe some of these alternatives.

Gradual migration take-up (M1) with this migration model, the operator decides to exploit the copper infrastructure to backhaul the indoor femto BSs. As a result, in the year 2011 the operator selects A2. Afterward, the mobile operator gradually migrates from A2 to A5. We assume that the operator starts replacing the copper infrastructure with a PON-based backhaul 3 years before the copper infrastructure is expected to be unable to support the required traffic levels. This occurs when the traffic demand for fixed broadband access networks exceeds 100 [Mbps] per household and is calculated using the traffic forecast model shown in [18]. In addition, the operator also gradually replaces the microwavebased backhaul used for the macro BSs with PONs. This process starts 3 years before the time when the area traffic demand is expected to exceed $1000\left[\mathrm{Mbps} / \mathrm{km}^{2}\right]$. During the migration process in the first year only $20 \%$ of the old technology is replaced, in the second year $50 \%$, in the third year $10 \%$, and by the fourth year $100 \%$ of the old technology has been replaced with the new one.

Gradual migration take-up (M2) similarly to the previous migration model, in M2 it is assumed that in the year 2011 the operator chooses A2. Afterward, the operator gradually migrates from A2 to A4. The copper infrastructure is gradually replaced with PON-based backhaul (as described in the migration model M1). On the other hand, in this case the operator keeps relying on the microwave-based backhaul for the macro BSs.

Immediate take-up (M3) with this migration model, the operator in 2011 decides to make a large investment replacing at once both the copper and the microwave infrastructure with PONs. Hence, in the year 2011 the operator migrates directly to A5.

Immediate take-up (M4) the M4 model is similar to M3, but in this case the operator only replaces the copper infrastructure with a PON-based backhaul, while keeping the microwave infrastructure for backhauling the macro BSs. As a result, in the year 2011 the operator migrates directly to A4.

No take-up (M5) this case is similar to M1, where the operator chooses A2 and then migrates to A5. However, in this case the migration is not carried out gradually. In fact, the operator keeps the VDSL2 copper infrastructure until its capacity reaches the exhaustion point (i.e., fixed broadband access networks capacity exceeds 100 [Mbps]) and only at that point the operator replaces it with a PON-based backhaul. Similarly, the replacement of the microwave-based backhaul with PONs is only carried out after the wireless traffic demand exceeds $1000\left[\mathrm{Mbps} / \mathrm{km}^{2}\right]$.

No take-up (M6) this migration model is almost the same as M2 with the only difference that the migration from copperbased to PON-based backhaul for the femto cells does not proceed gradually. On the other hand the macro BSs are always backhauled via microwave.

The value of the TCO is evaluated for all the migration options described above to find the most economically 
Table 2 Inputs for power consumption and investment costs [4, 8, 1720]

\begin{tabular}{llr}
\hline Eq./comp & $\mathrm{P}[\mathrm{W}]$ & Cost Eq./service [USD] \\
\hline DSLAM & 85 & 1750 \\
GES & 50 & 2400 \\
CES & 300 & 3000 \\
Hub switch & 53 & 2930 \\
Antenna & 37 or 92,5 & 4472 \\
OLT & 105 & 3000 \\
Splitter (32 ports) & 0 & 140 \\
ONU & 4 & 146 \\
VDSL2 modem & 5 & 30 \\
SFP & 1 & 37 \\
SFP + & 1,5 & 78 \\
Fiber $[\mathrm{km}]$ & - & 160 \\
Trenching $[\mathrm{km}]$ & - & 130,000 \\
\hline
\end{tabular}

attractive. The power consumption and cost values shown in Table 2 are used in our calculations.

With respect to the microwave backhaul technology, it is assumed that the microwave antenna power consumption corresponds to $P_{\text {low-c }}$ when the traffic at the antenna is lower than 500 [Mbps], and to $P_{\text {high-c }}$ when the traffic is higher. Fast Ethernet connections operating at $100[\mathrm{Mbps}]$ are used inside the buildings to connect the femto BSs to the GES (in A1 and A3). In addition, the femto BSs are distributed uniformly among the buildings and in the area. As a result, the number of GESs $\left(N_{\mathrm{GES}}\right)$ is equal to the number of buildings if $\eta>0$.
We assume that the power consumption of a GES linearly scales with the number of ports that are used for backhauling the femto BSs, i.e., $P_{\mathrm{GES}}=\frac{N_{\text {femto }}}{N_{\mathrm{b}} n_{\text {ports }}^{\mathrm{GES}}} P_{\mathrm{GES}}^{\max }, \forall \eta>0.1$, where $P_{\mathrm{GES}}^{\max }$, and $n_{\text {ports }}^{G E S}$ are the maximum power of a GES and the total number of ports of the GES, respectively. Moreover, in the Greenfield scenario, two possible values are considered for the maximum distance between the femto BSs and the local exchanges, i.e., $300[\mathrm{~m}]$ and $1[\mathrm{~km}]$. On the other hand, in the Brownfield scenario, we consider only a maximum distance of $300[\mathrm{~m}]$ between the femto BSs and the local exchanges. This is because VDSL2 technologies are not able to cope with distances longer than 300 [m], i.e., A2 cannot be considered as a migration option.

For this study, a technician salary is assumed to be $72[\mathrm{USD} / \mathrm{h}]$ for the first year and the energy cost is set to $0.15[\mathrm{USD} / \mathrm{kWh}]$. After the first year we assume a yearly increase based on the geometric progression $c_{n}=c_{1} q^{n-1}$, where $c_{n}$ represents either a technician salary or the energy cost in the year $n$ and $q=1,03$ is the increase ratio [4]. Unless stated differently, a fixed yearly depreciation of $5 \%$ is also specified for the network equipment cost.

\section{Results}

This section presents the numerical results obtained for the case study described before. It is divided into two parts, i.e., Greenfield and Brownfield considerations.

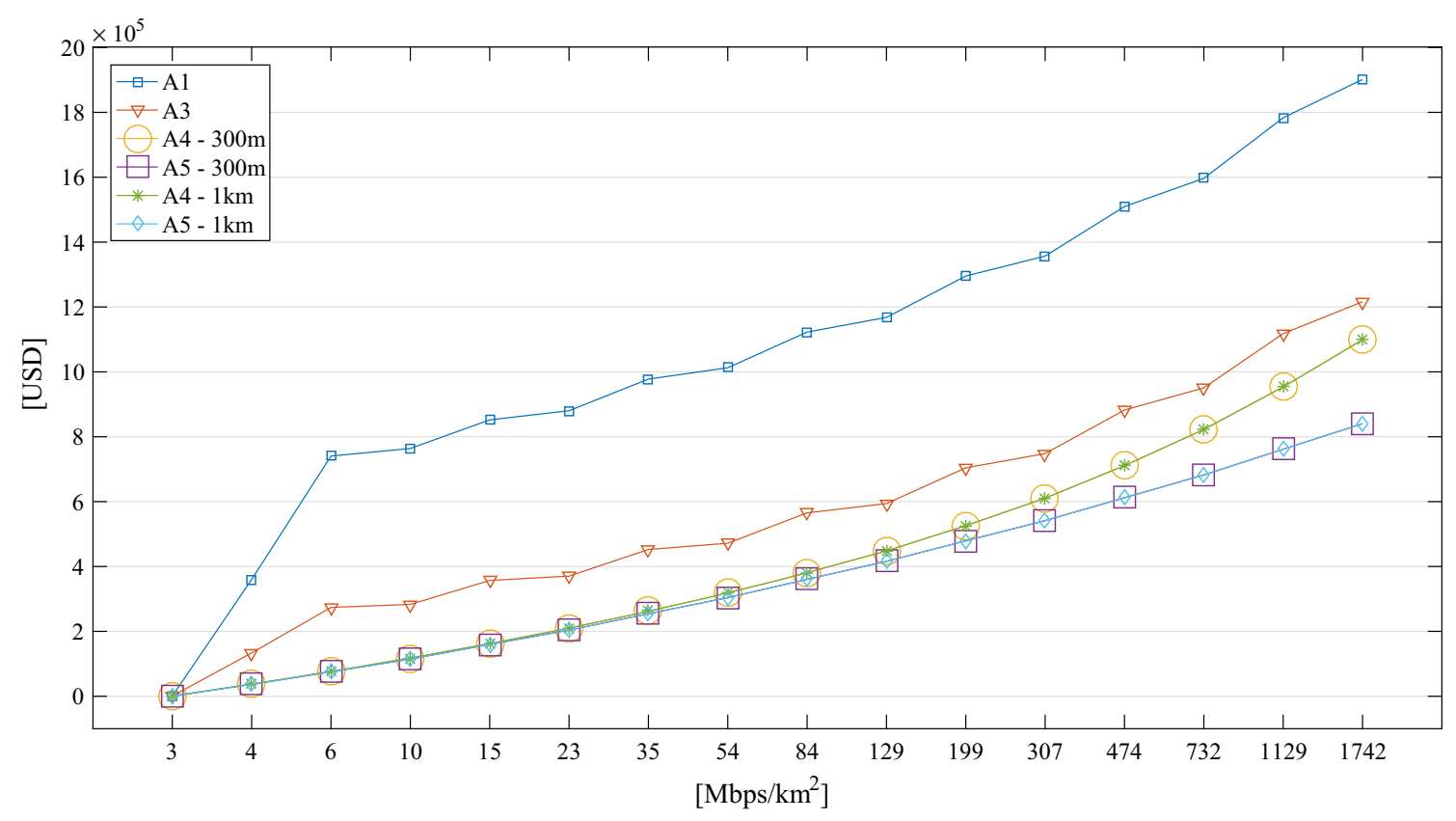

Fig. 4 Energy consumption cost of the proposed backhaul architectures in a Greenfield scenario as a function of the area traffic demand values from year 2010 until year 2025 


\subsection{Greenfield scenario}

Figure 4 illustrates the total energy consumption cost as a function of the area traffic demand considering A1, A3, $\mathrm{A} 4$, and A5. When A1 and A3 are compared, it is evident that using a fiber-based backhaul for femto BSs can significantly reduce the overall energy cost. These savings become even higher when FTTH solutions (A4 and A5) are employed. More specifically, the best results are obtained using a PON-based backhaul for both femto and macro BSs, i.e., A5. Moreover, it can be observed that the energy cost in the scenario where the maximum distance between a local exchange and a femto BS is $300[\mathrm{~m}]$ is the same as in the case where the maximum distance is $1[\mathrm{~km}]$ (even if the total number of required local exchanges is higher when the maximum distance is set to $300[\mathrm{~m}])$. This is because the energy consumption of a local exchange with the above-mentioned architectures is negligible. In A4 and A5 they are equipped with passive components, while in A3 they do not have any fiber switching component at all. As a result in Fig. 4 the curve for "A5-300 m" and the curve for "A5-1 km" overlap. The same happens to the curve for "A4-300 m" and the curve for "A4-1 km."

Figure 5a illustrates the average energy consumption cost, per user, per year. It can be observed that using FTTH and FTTB architectures for backhauling the femto BSs leads to a significant reduction in the energy cost with respect to the microwave-based solution. In particular, when A3 is used, it is possible to save up to 1.57 [USD/user/year] with respect to $\mathrm{A} 1$, while with $\mathrm{A} 5$ it is possible to save up to 2.16 [USD/user/year]. These savings are slightly higher than the ones that can be obtained with A4.

Figure $5 \mathrm{~b}, \mathrm{c}$ provides a more complete picture of the cost analysis of the various architectures by showing the TCO for a period $T$ of 15 years.

The results presented in Fig. 5b are based on the assumption that the operator builds a new infrastructure, which means that for A3, A4, and A5 the operator performs a large investment for trenching the fiber cables to connect the radio BSs to the MN. The figure shows that, due to this large investment, A3, A4, and A5 show a higher TCO than A1 (up to 16.13 [USD/user/year] higher in the case of A4). In fact, A4 is the most expensive and has a TCO as high as 58.48 [USD/user/year].

The results in Fig. $5 \mathrm{c}$ are obtained by assuming that when deploying A3, A4, and A5, the operator leases dark fiber from a third-party entity (e.g., an infrastructure operator) to connect the radio BSs to the MN. Leasing dark fiber is an option to decrease CAPEX by avoiding the high investment required for fiber trenching. It also ensures faster deployment time. In this case, a contract is signed between the operator and the third-party entity, where the operator pays a yearly fee in return for the fiber connectivity.

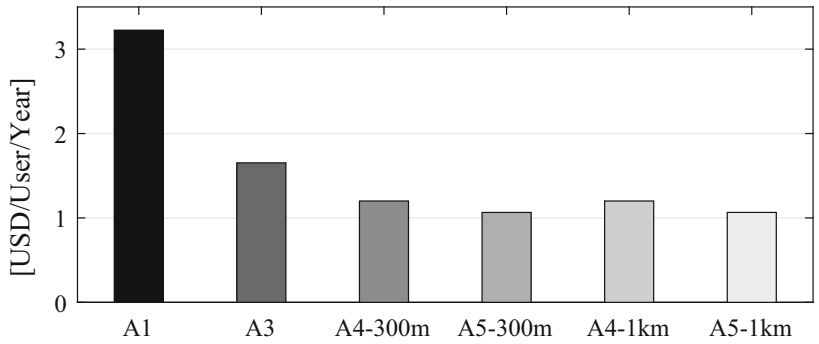

(a)

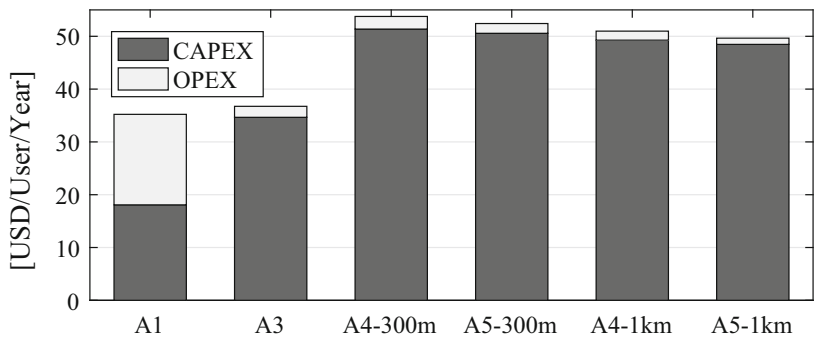

(b)

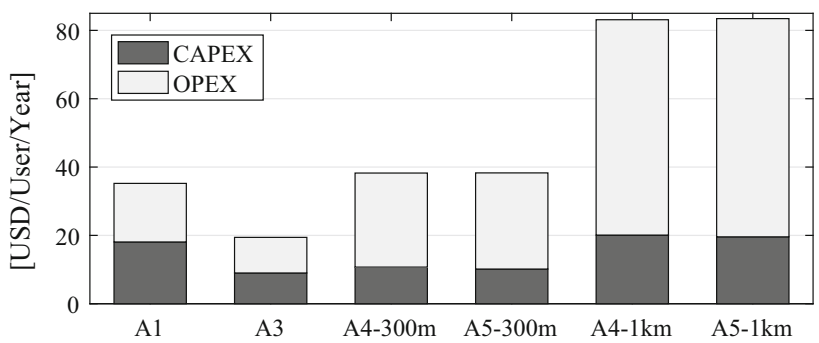

(c)

Fig. 5 Energy consumption cost and TCO values for the proposed backhaul architectures in a Greenfield scenario over a time period $T$ equal to 15 years: a energy cost per user per year, b TCO assuming trenching, $\mathbf{c}$ TCO assuming leasing dark fibers

Depending on the architecture, the dark fiber leasing might or might not be a good alternative. Figure $5 \mathrm{c}$ shows that leasing the dark fiber in $\mathrm{A} 3$ is the most attractive solution since it leads to the lowest TCO among all the considered cases (i.e., 15.8 [USD] lower than A1). On the other hand, A4 and A5 become hardly feasible in terms of costs when the maximum distance between the femto BSs and the local exchanges is $1[\mathrm{~km}]$. In fact, the results show that with these two architectures leasing dark fibers, besides triggering a reduction of CAPEX, has also the unexpected effect of increasing significantly the value of OPEX, up to a point in which a solution based on leasing fiber resources becomes even more expensive than trenching a fiber infrastructure. This is because of the higher distance between the local exchange and the endusers (i.e., $1[\mathrm{~km}]$ instead of $300[\mathrm{~m}]$ ) that requires the leasing of more fiber in the network.

\subsection{Brownfield scenario}

Figure 6 shows the energy consumption cost of the different migration alternatives described in Sect. 5. These values 


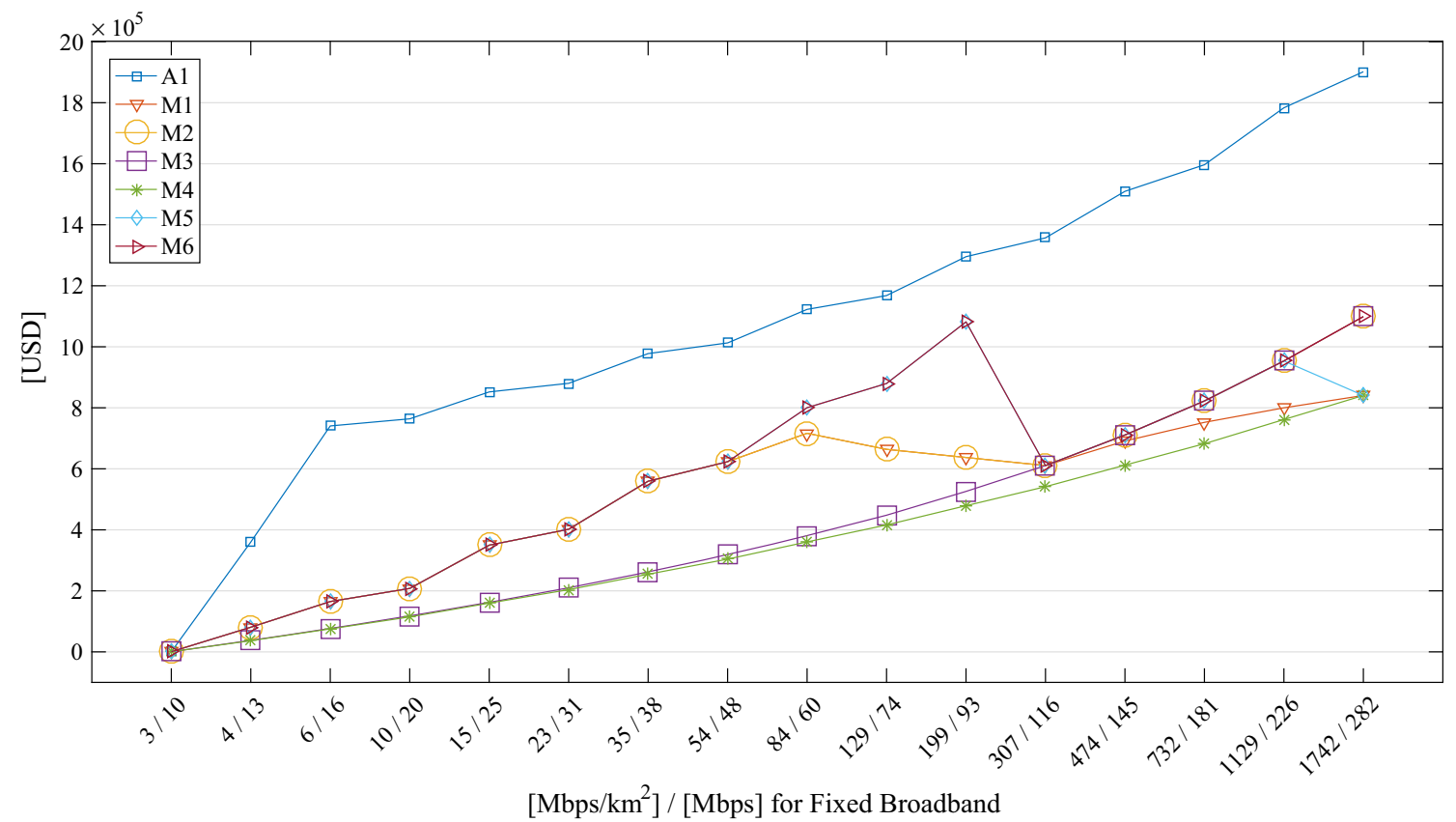

Fig. 6 Energy consumption cost of each of the migration options in the Brownfield scenario as a function of the area traffic demand from year 2010 until year 2015. The horizontal axis reports also, for any given year, the value of the minimum amount of capacity that a fixed broadband segment should be able to offer according to the EU digital agenda requirements are reported as a function of the area traffic demand. The horizontal axis reports also, for a given year, the value of the minimum amount of capacity that a fixed broadband segment should be able to offer according to the EU Digital Agenda requirements [18]. This latter value is important to understand when a copper-based broadband segment will reach its exhaustion point, i.e., a value of capacity greater or equal than 100 [Mbps]. It can be observed that the immediate takeup approaches (M3 and M4) are the most energy-efficient solutions. On the other hand, the migration options that employ no take-up (M5 and M6), i.e., that exploit existing copper infrastructure for backhaul until the copper capacity reaches its exhaustion point, are the least energy efficient. This is because M5 and M6 keep using a copper-based infrastructure for a longer period of time. Note that the curves corresponding to M5 and M6 overlap in all but one case (i.e., after $1129\left[\mathrm{Mbps} / \mathrm{km}^{2}\right]$ ), while the curves corresponding to M3 and M4 overlap up to $84\left[\mathrm{Mbps} / \mathrm{km}^{2}\right]$. Moreover, the energy consumption performance when using gradual take-up strategies (M1 and M2) lies in the middle of the previous cases. M1 and M2 solutions perform better in terms of energy cost with respect to M5 and M6 because the VDSL2 infrastructure begins to be replaced at an earlier point in time (i.e., 3 years before the capacity of copper is exhausted). Finally, it is evident from Fig. 6 that all the considered migration strategies (M1-M6) present a lower energy cost than the backhaul solution that is based on microwave only (i.e., A1). In fact, using microwave links for backhauling the indoor femto BSs leads to a higher energy consumption than using any fixed broadband infrastructure (i.e., based on copper or fiber).

Figure 7a shows the energy cost per user per year. The results show that among the Brownfield migration alternatives, M3 is the most energy efficient since it leads to an energy cost of 1.06 [USD/user/year]. This low cost is due to the fact that the upgrade toward PON (i.e., A5) already occurred in the year 2011, i.e., when indoor BSs are first deployed.

Figure $7 \mathrm{~b}$ presents the TCO values for the proposed Brownfield migration alternatives. Remember that in our study we take into account a fixed yearly depreciation for the network equipment cost of 5\%. It can be seen that the migration options based on immediate take-up (M3 and M4) are the most expensive due to the high initial investment costs (which translates into high CAPEX values). However, the migration options based on gradual take-up (M1 and M2) and those based on no take-up (M5 and M6) show very similar costs and represent the least expensive alternatives in terms of cost per user per year. This proves that using the legacy copper infrastructure close to (or up to) the capacity exhaustion may bring significant financial benefits for an operator. However, it should be noted that M5 and M6 have the slightly higher OPEX values because they present higher energy, maintenance, and fault management costs.

Figure $7 \mathrm{c}$ shows the TCO values for the different migration strategies when considering a yearly depreciation rate for the 


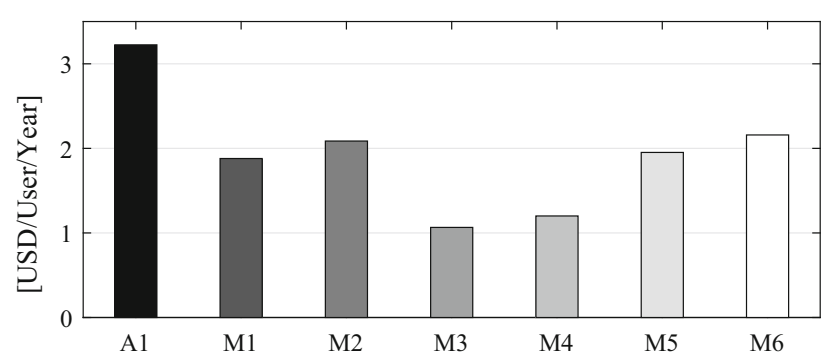

(a)

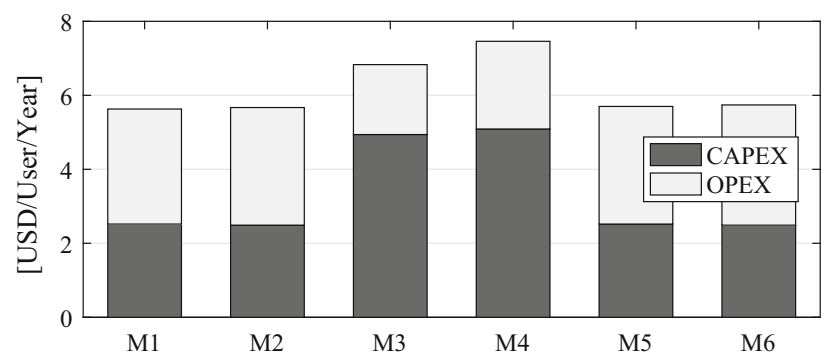

(b)

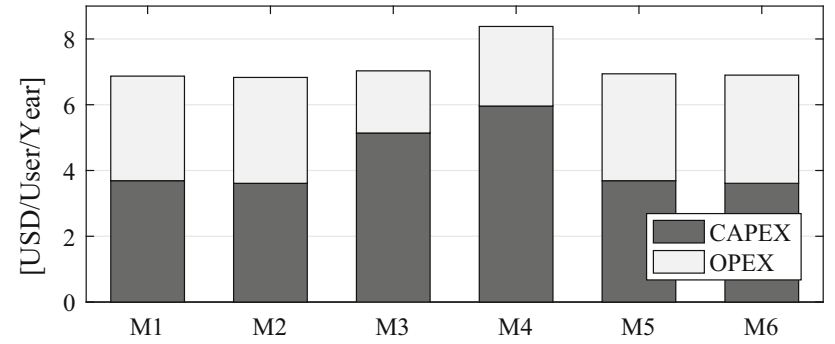

(c)

Fig. 7 Energy consumption cost and TCO values for the migration options of the Brownfield scenario over a time period T equal to 15 years: a energy consumption per user per year, $\mathbf{b} \mathrm{TCO}$ values assuming a yearly depreciation of the equipment cost of $5 \%$, $\mathbf{c}$ TCO values assuming a yearly depreciation of the equipment cost of $2.5 \%$

network equipment of $2.5 \%$. It can be seen that the trends are similar to those in Fig. 7b, even if the relative difference between the different solutions is slightly smaller.

Our results show that exploiting the legacy copper infrastructure leads to a reduction in costs for the operators and the M1 and M2 migration models are the most economically beneficial.

\subsection{Sensitivity analysis}

This section presents a sensitivity analysis with the objective of validating the results presented in previous section. The backhaul technologies are divided into three categories: copper, fiber, and microwave. The most significant cost parameter (i.e., the one having the largest impact on the TCO) is determined for each category. This parameter is then varied in the range between 70 and $130 \%$ with respect to its original value (shown in Table 2), and the corresponding impact on the TCO is evaluated.

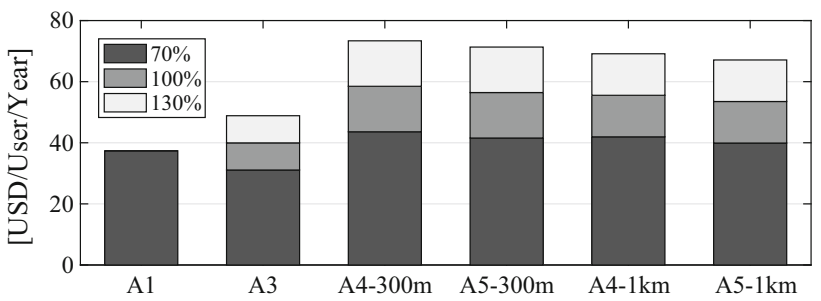

Fig. 8 TCO values of the proposed backhaul architectures obtained varying the fiber trenching cost between 70 and $130 \%$ of its original value (Table 2)

With respect to copper-based backhaul solutions, the most relevant TCO parameter is the cost of the DSL modem (categorized as Equipment cost in Fig. 3). The parameter is varied between 70 and $130 \%$ of its original value (reported in Table 2), and no significant changes are detected in the total TCO value of the copper-based backhaul architectures. As a result, it can be concluded that varying the DSL modem cost does not affect the conclusions drawn in the previous sections.

With respect to fiber-based backhaul solutions, the most relevant TCO parameter depends on whether the operator is deploying its own fiber infrastructure or is leasing dark fibers. In the former case, the most relevant TCO parameter is the fiber trenching cost (categorized as Infrastructure cost in Fig. 3). Figure 8 shows the results obtained by varying the fiber trenching cost between 70 and $130 \%$ of its original value reported in Table 2 . It is clear that when the fiber trenching cost is reduced, A3 becomes the most attractive solution in financial terms (A3 becomes more cost efficient than A1 when the fiber trenching cost is reduced below $91 \%$ of its original value). However, A4 and A5 always remain the most expensive alternatives. Moreover, increasing the fiber trenching cost does not affect the conclusions drawn in the previous sections.

If the operator leases the dark fiber, the most relevant TCO parameter is the fiber leasing cost (categorized as Spectrum and fiber leasing cost in Fig. 3). Figure 9 shows the results obtained by varying the fiber leasing cost between 70 and $130 \%$ of its original value (shown in Table 2). It can be noted that reducing the fiber leasing cost does not significantly affect the conclusions drawn in the previous sections. In fact, A3 remains the most attractive backhaul solution. The only observable change is that A5 is found to be more cost efficient than A1 if the fiber leasing cost is reduced to $72 \%$ of its original value (but only in case when the distance between the femto BSs and local exchanges is at most 300 [m]).

With respect to the microwave-based backhaul solutions, there are two cost parameters that have a large impact on the TCO. The first one is the cost of the microwave antenna (categorized as Equipment cost in Fig. 3). We varied this parameter between from 70 and $130 \%$ of its original value, and the 


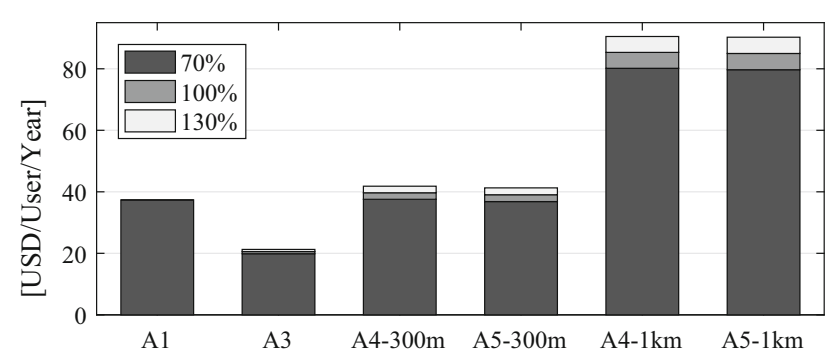

Fig. 9 TCO values of the proposed backhaul architectures obtained varying the dark fiber leasing cost between 70 and $130 \%$ of its original value (Table 2)

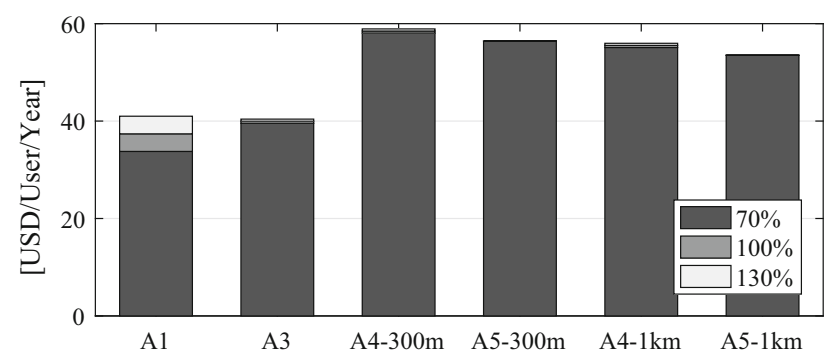

(a)

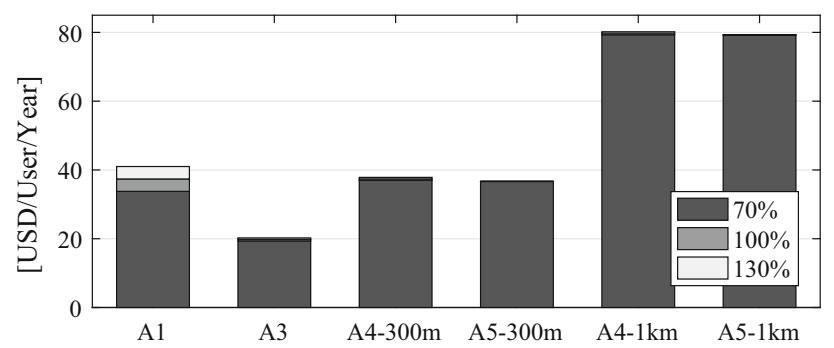

(b)

Fig. 10 TCO values of the proposed backhaul architectures obtained varying the cost of the microwave antenna between 70 and $130 \%$ of its original value (Table 2). Two cases are presented: a The operator trenches and owns the fiber infrastructure, $\mathbf{b}$ the operator leases dark fibers

corresponding impact on the TCO is shown in Fig. 10. In particular, Fig. 10a presents the case when the operator trenches the fiber infrastructure. The figure shows that decreasing the microwave antenna cost does not change the conclusions drawn in previous section and that A1 remains the most costefficient solution. However, increasing the antenna cost to $126 \%$ of its original value makes A3 the most attractive solution from an economical standpoint. Figure $10 \mathrm{~b}$ presents the case when the operator leases dark fibers. The trends are similar to the one in Fig. 10a. More specifically, reducing the cost of the microwave antenna does not affect the results and A3 remains the most cost-efficient solution. However, increasing the microwave antenna cost over $103 \%$ of its original value makes A1 more expensive than A5 (in case when the distance between the femto BSs and local exchanges is at most $300[\mathrm{~m}]$ ). In addition, increasing the antenna cost over

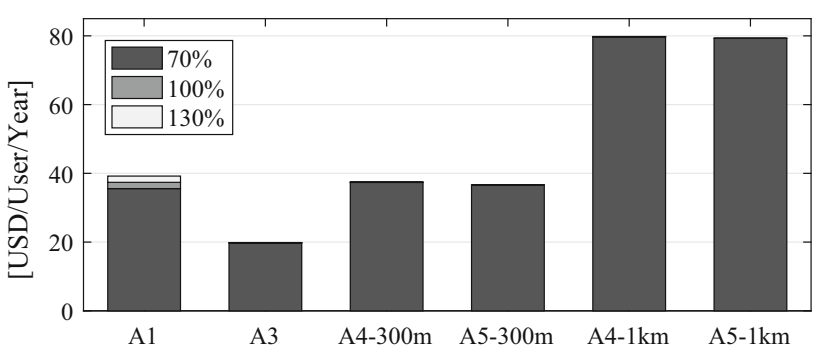

Fig. 11 TCO values of the proposed backhaul architectures obtained varying the microwave spectrum cost between 70 and $130 \%$ of its original value (Table 2)

$124 \%$ of its original value also makes A4 more cost efficient than A1.

The second TCO parameter whose variation is critical in a microwave-based backhaul is the spectrum leasing cost (categorized as Spectrum and fiber leasing cost in Fig. 3). Figure 11 shows the TCO values of the proposed backhaul architectures obtained by varying the spectrum leasing cost between 70 and $130 \%$ of its original market value. Only the case with fiber leasing was taken into account because in the case with fiber trenching the conclusions drawn in the previous sections remain unchanged. Figure 11 shows that reducing the spectrum cost does not affect the overall conclusions already discussed so far, i.e., A3 remains the most cost-efficient option. On the other hand, increasing the spectrum cost over $28 \%$ of its original value makes A5 more cost-effective than A1 (in case when the distance between the femto BSs and local exchanges is at most $300[\mathrm{~m}]$ ).

Based on the sensitivity analysis results discussed so far we can state that changing the cost of the parameters that have largest impact on the TCO of the proposed backhaul architectures did not significantly affect the overall conclusions drawn in the previous sections. However, it was observed that the costs of fiber trenching and of the microwave antenna are the most sensitive since a relatively small change can bring significant differences in the relative TCO values of some backhaul architectures.

\section{Conclusions}

This article proposed a total cost of ownership (TCO) assessment methodology that can be used to evaluate the TCO performance of backhaul networks. The methodology includes four steps: (i) wireless traffic demand estimation, (ii) wireless network deployment, (iii) backhaul network deployment (for Greenfield scenarios) or backhaul network migration (for Brownfield scenarios), and (iv) TCO evaluation. The TCO assessment methodology was employed in a Greenfield scenario to compare the TCO values of four backhaul network architectures and in a Brownfield scenario to 
compare the TCO values of six backhaul network migration options. In both cases a wireless network composed of indoor femto and outdoor macro BSs was considered.

The analysis of the Greenfield scenario proves that backhaul architectures based on PONs are very energy efficient, but they also require a large investment (due to trenching or leasing of the fiber infrastructure) that leads to high TCO values. This is particularly true when the distance between femto BSs and local exchanges is long (e.g., greater or equal to $1[\mathrm{~km}])$. Our results prove that using a fiber to the building (FTTB) infrastructure for backhauling femto BSs and a microwave links for backhauling macro BSs becomes the most cost-efficient solution when it is possible to lease dark fiber already deployed in the area.

The analysis of the Brownfield scenario shows that from an energy consumption perspective the best solution is to migrate toward PONs as early as possible (i.e., migration options M3 and M4). However, this also results in very high TCO values. A more cost-efficient solution is to exploit the existing copper infrastructure for backhauling the indoor femto cells and gradually replace it starting from a few years before the copper capacity exhaustion point (i.e., migration options M1 and M2).

Finally, we plan to extend the study presented in the paper and to apply the proposed assessment methodology to evaluate the impact that spatial and temporal traffic variations have on the TCO value of a mobile network (i.e., radio + transport). In this regard we also plan also to investigate potential dynamic energy management schemes specifically tailored for the proposed architectures.

Open Access This article is distributed under the terms of the Creative Commons Attribution 4.0 International License (http://creativecomm ons.org/licenses/by/4.0/), which permits unrestricted use, distribution, and reproduction in any medium, provided you give appropriate credit to the original author(s) and the source, provide a link to the Creative Commons license, and indicate if changes were made.

\section{References}

[1] Fiorani, M., Tombaz, S., Farias, F.S., Wosinska, L., Monti, P.: Joint design of radio and transport for green residential access networks. In: IEEE Journal on Selected Areas of Communications (JSAC) Special Issue on Energy-Efficiency in 5G (2016)

[2] Tombaz, S., Monti, P., Wang, K., Vastberg, A., Forzati, M., Zander, J.: Impact of backhauling power consumption on the deployment of heterogeneous mobile networks. In: IEEE Global Communication Conference (GLOBECOM) (2011)

[3] Monti, P., Tombaz, S., Wosinska, L., Zander, J.: Mobile backhaul in heterogeneous network deployments: Technology options and power consumption. In: IEEE International Conference on Transparent Optical Networks (ICTON), pp. 1-7 (2012)

[4] Mahloo, M., Monti, P., Chen, J., Wosinska, L.: Cost modeling of backhaul for mobile networks. In: IEEE International Conference on Communications Workshops (ICC), pp. 397-402 (2014)
[5] Mesodiakaki, A., Adelantado, F., Antonopoulos, A., Kartsakli, E., Alonso, L., Verikoukis, C.: Energy impact of outdoor small cell backhaul in green heterogeneous networks. In: IEEE International Workshop on Computer Aided Modeling and Design of Communication Links and Networks (CAMAD), pp. 11-15 (2014)

[6] Tombaz, S., Monti, P., Farias, F., Fiorani, M., Wosinska, L., Zander, J.: Is backhaul becoming a bottleneck for green wireless access networks?. In: IEEE International Conference on Communications (ICC), pp. 4029-4035 (2014)

[7] Widaa, A.A., Markendahl, J., Ghanbari, A.: Toward capacityefficient, cost-efficient and power-efficient deployment strategy for indoor mobile broadband. In: European Regional Conference of the International Telecommunications Society (2013)

[8] Ahmed, A.A.W., Markendahl, J., Cavdar, C., Ghanbari, A.: Study on the effects of backhaul solutions on indoor mobile deployment macrocell vs. femtocell. In: IEEE International Symposium on Personal Indoor and Mobile Radio Communications (PIMRC), pp. 2444-2448 (2013)

[9] Frias, Z., Pérez, J.: Techno-economic analysis of femtocell deployment in long-term evolution networks. EURASIP J. Wirel. Commun. Netw. 1, 1-15 (2012)

[10] Coldrey, M., Berg, J.E., Manholm, L., Larsson, C., Hansryd, J.: Non-line-of-sight small cell backhauling using microwave technology. IEEE Commun. Mag. 51, 78-84 (2013)

[11] Breuer, D., Krauss, S., Geilhardt, F., Weis, E., Belschner, J.: Cost evaluation of small cell backhaul architectures. In: Conference of Telecommunication, Media and Internet Techno-Economics (CTTE), pp. 1-5 (2015)

[12] Suarez, L., Bouraoui, M.A., Mertah, M.A., Morvan, M., Nuaymi, L.: Energy efficiency and cost issues in backhaul architectures for high data-rate green mobile heterogeneous networks. In: IEEE Annual International Symposium on Personal, Indoor, and Mobile Radio Communications (PIMRC), pp. 1563-1568 (2015)

[13] Cioffi, J.M.: Lighting up copper [History of Communications]. IEEE Commun. Mag. 49, 30-43 (2011)

[14] Earth Project: Energy efficiency analysis of the reference systems, areas of improvements and target breakdown. Deliverable D2.3 (2010)

[15] Senza Fili Consulting: Crucial economics for mobile data backhaul. White paper, (2011)

[16] Bern, M.W., Graham, R.L.: The shortest network problem. Sci. Am. J. 260, 84-89 (1989)

[17] Cisco: Cisco visual networking index: global mobile data traffic forecast update, 2015-2020. White paper (2016)

[18] Optical Access Seamless Evolution (OASE) Project: Migration paths. Deliverable D3.4 (2013)

[19] Digital Subscriber Line warehouse: Copper-based equipment prices. http://www.dsl-warehouse.com/

[20] Data interfaces shop: Equipment prices. http://www. datainterfaces.com

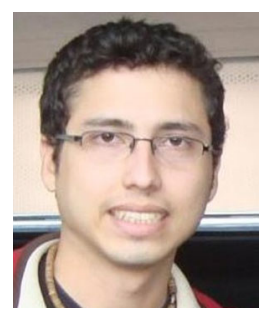

Fabricio Farias received the Ph.D. degree from the Universidade Federal do Pará (UFPA), Brazil, in 2016. He is assistant professor with UFPA-Campus Cametá since 2014. His research interests include data mining, DSL networks, mobile backhaul, digital $\mathrm{TV}$, and heterogeneous networks. 


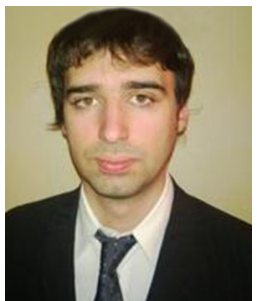

Matteo Fiorani is Postdoc Researcher at KTH Royal Institute of Technology (Sweden) since January 2014. He was visiting researcher in August/September 2015 at University of California, Davis, CA (USA), and April/May 2016 at Columbia University, New York, NY (USA). He holds a Ph.D. in Information and Communication Technologies (ICT) from University of Modena (Italy). During his Ph.D. studies he spent 1 year at Vienna University of Technology (Austria). Dr. Fiorani's research interests are: (i) 5G radio and transport technologies, (ii) optical and electronic interconnects for data centers, (iii) software-defined networking (SDN). He co-authored more than 40 peer-reviewed research papers (18 journal papers). He is currently involved in several European and Swedish research projects. He is co-founder and chair of the IEEE workshop on $5 \mathrm{G}$ transport networks (5GT).

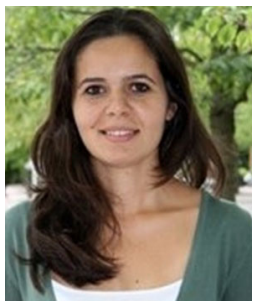

Sibel Tombaz received her B.S. (with highest honors) degree in electrical and electronics engineering from Istanbul University, Turkey, in 2006. In January 2009 she got her M.S. degree in electrical and electronics engineering from the same university. From March to October 2008 she was a visiting scholar at the WCSP group, University of South Florida, Tampa. She received her $\mathrm{Ph} . \mathrm{D}$. degree at the Communication Systems Department under the guidance of Prof. Jens Zander, KTH Royal Institute of Technology, Stockholm, Sweden. She is currently working as a Senior Researcher at Ericsson, Stockholm, Sweden. Her research interests include 5G networks, mmWave communication, MIMO, beamforming schemes, backhaul/fronthaul technologies, and energy efficiency improvements of wireless networks.

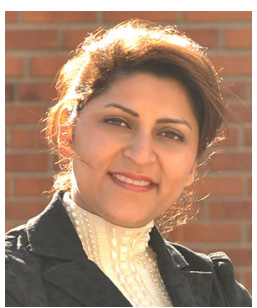

Mozhgan Mahloo received her Ph.D. degree from KTH Royal Institute of Technology, Sweden, in 2015. Currently, she is working as Experienced Researcher in Ericsson AB in the field of Cloud Technologies. Her research interests are technology performance analysis in the ICT world with a focus on the cost and reliability assessment.

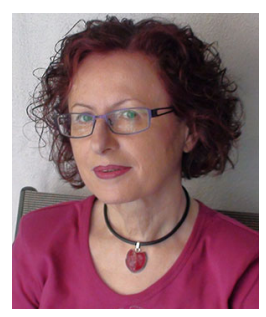

Lena Wosinska received her Ph.D. degree in Photonics and Docent degree in Optical Networking from KTH Royal Institute of Technology, Sweden, where she is currently a Full Professor in Telecommunication in the School of Information and Communication Technology (ICT). She is founder and leader of the Optical Networks Lab (ONLab). She has been working in several EU projects and coordinating a number of national and international research projects. Her research interests include fiber access and $5 \mathrm{G}$ transport networks, energy-efficient optical networks, photonics in switching, optical net- work control, reliability and survivability, and optical data center networks. She has been involved in many professional activities including guest editorship of IEEE, OSA, Elsevier and Springer Journals, serving as General Chair and Co-Chair of several IEEE, OSA and SPIE conferences, and workshops, serving in TPC of many conferences, as well as being reviewer for scientific journals and project proposals. She has been an Associate Editor of OSA Journal of Optical Networking and IEEE/OSA Journal of Optical Communications and Networking. Currently she is serving on the Editorial Board of Springer Photonic Networks Communication Journal and of Wiley Transactions on Emerging Telecommunications Technologies.

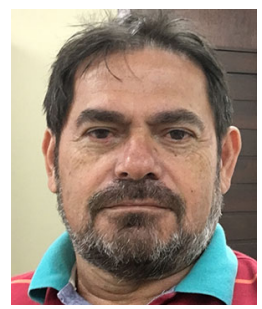

Joao C. W. A. Costa Albuquerque Costa received the Ph.D. degree in Electrical Engineering and Telecommunications from the State University of Campinas (UNICAMP), in 1994. Currently he is professor at the Faculty of Computer and Telecommunication Engineering of the Institute of Technology. $\mathrm{He}$ is the author of more than 80 refereed papers in journals and co-author of two international patents. He has supervised 28 master students, 14 Ph.D. thesis, and about 100 undergraduate students.

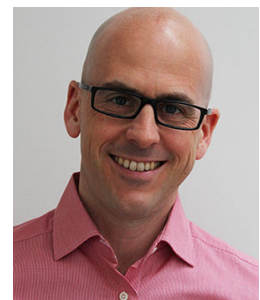

Paolo Monti is an Associate Professor at KTH Royal Institute of Technology. He received a Ph.D. degree (2005) from the University of Texas at Dallas where he then worked as a Research Associate until 2008. Dr. Monti serves on the Editorial Boards of the IEEE Transactions on Green Communications and Networking and of the Springer Photonic Network Communications Journal. He co-authored more than 100 technical papers, with three best paper awards. Dr. Monti regularly participates in the TPCs of the main IEEE conferences. He co-chaired, among others, one workshop on network survivability (ICC 2012) and four workshops on green broadband access (ICC 2013, Globecom 2014, ICC 2015, and ICC 2017). Dr. Monti was also the TPC chair of IEEE ONDM 2014 and a TPC Co-Chair of the Symposium on Optical and Grid Computing at IEEE ICNC 2014 and 2016. He is currently a TPC Co-Chair of IEEE OnlineGreenComm 2016, of HPSR 2017, of the ONS symposium at IEEE Globecom 2017, and of the OGN symposium at ICNC 2017. His main research interests are within the networking aspects of all-optical networks with a focus on transport solutions (i.e., covering both data and control plane) for next-generation 5G networks. Dr. Monti is a Senior Member of IEEE. 